\title{
Optimized Joint Coding Algorithm for Audio Streaming in Short Range Wireless Networks
}

\author{
Jelena Kovacevic ${ }^{1}$, Dragan Samardzija ${ }^{2}$ and Miodrag Temerinac ${ }^{3}$ \\ ${ }^{1}$ Faculty of Technical Sciences, University of Novi Sad, Trg Dositeja Obradovica 6, 21000 Novi Sad, Serbia \\ ${ }^{2}$ Bell Laboratories, Alcatel-Lucent, L-273, 791 Holmdel-Keyport Road Holmdel, NJ 07733, USA \\ ${ }^{3}$ Micronas GmbH, Hans-Bunte Strasse 19 D-79108 Freiburg, Germany
}

\begin{abstract}
Streaming high quality audio/video (AV) from home media sources to TV sets and audio speakers over a wireless local area network (WLAN) is a challenging problem because of the fluctuating bandwidth caused by interference and fading. Retransmission and buffers as common techniques in data transfer over error prone channel are not adequate for delay sensitive applications such as high quality audio streaming. Our approach is to adjust the audio bit-rate dynamically in order to improve the perceptual quality of audio according to wireless channel quality. In this paper we specifically focus on robust audio streaming over short range ISM-band wireless channels. The major strength of the proposed algorithm is in its ability to match the source and channel coding parameters to given channel conditions, thus providing better quality of audio in home networking.
\end{abstract}

\section{INTRODUCTION}

Wireless networks are becoming a key enabler to a rapidly growing number of consumer applications. Short range wireless communication systems are of a particular interest. For example audio and video streaming, gaming as well as integrated multimedia entertainment centers rely heavily on wireless communications. In recent years a number of short range wireless communication standards has emerged targeting consumer applications (802.11a, b and $n$, UWB ...). In addition, a number of proprietary solutions are being developed (WirelessHD, WHDI...).

Unlike the data applications, audio and video are intolerant of bandwidth fluctuations due to the delay constraints. Guaranteed bandwidth and QoS are essential requirements in order to satisfy customer expectations for "wire-like" performance. Assuring high bandwidth is essential but not sufficient. When several applications try to access the same bandwidth, the ones that are intolerant to time delays and bandwidth fluctuations will not function properly. MAC layer and cross layer optimization is well investigated [1]. However, methods for providing good quality of audio in such environments are open issue.

What we propose is an optimization algorithm in a source-channel domain. Since the early days of digital communications, channel coding and modulation have been separated from source coding. According to the information theoretic results optimal source and channel coding can be performed separately for the stationary additive white Gaussian noise channel (AWGN), when no latency constraint is imposed. The result is known as Separation Theorem [2].

Audio and video streaming (including broadcast and unicast services) over time-varying wireless channels and finite block data lengths (implicitly latency-constrained) cannot be optimally transmitted when source and channel coding are separated.

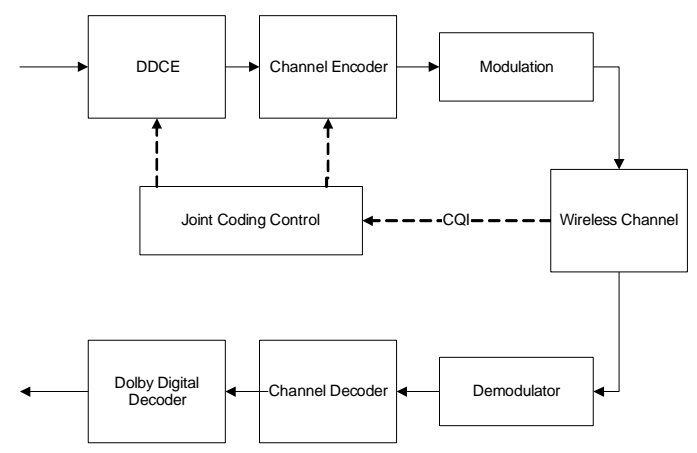

Fig. 1 Functional block diagram of the wireless communication system for audio multichannel streaming.

Current audio technologies (DTS, Dolby) relay on know channel capacity. To address the above problem in this study we consider a practical communication system for audio streaming that is depicted in Fig. 1. The transmitter consists of the multi channel audio encoder, channel encoder, modulator and joint coding control. Parameters of source and channel coding are set dynamically depending on the channel quality (quantified by the channel quality indicator (CQI)) and the size of the data block that is to be transmitted.

\section{JOINT CODING CONTROL}

To achieve a good perceptual quality, audio encoder relies on higher data rates (up $640 \mathrm{kbps}$ for the 5.1 format). This approach performs well in a case of wired channel or good wireless channel conditions (e.g., a short distance to the access point and low level of interference). Unlike the above case, poor channel conditions lead to lower throughput, higher frame error rate and consequently to a lower perceptual quality. In this paper, we propose joint coding control algorithm that optimize the transmission data rate according to the channel quality, thus keeping the perceptual quality as high as the channel allows. Basic idea of the proposed rate control algorithm is to increase the quantization noise of the source when channel bandwidth decreases, which will result in a smaller source bit-rate. Quantization noise could be seen as a smart error of the source which will decrease perceptual quality of audio, but on the other hand unpredictable error of the channel will lead to frame loss and audible artifacts of reproduced audio. If channel conditions are good, in our approach we will allow maximum source bit-rate (minimum quantization 
error) opposed to predefined bit-rate in traditional approach. The following algorithm, i.e., method is investigated:

Network Adaptive Rate Controller - The transmission rates of the source and channel encoders are dynamically matched according to the wireless channel quality. The adaptation is performed once per frame. Quantization noise is chosen to mach maximum throughput of the channel and adaptation rate is investigated. History of channel quality through certain amount of time $(\sim 1 \mathrm{~s})$ is used to determine how fast source data rate could be increased if channel conditions allow it. Decrease of source data rate has to be instantaneous.

The above method is compared to conventional source and channel coding. As the performance reference we consider the transmitter which consists of the audio encoder, channel encoder, modulator (without joint coding control and the constant transmission data rate).

\section{METHODOLOGY}

\section{A. Audio quality}

Audio quality measurements are used to assess the performance of the above system for different joint coding control methods and radio channel conditions. The following criteria are used:

PSNR and SNR - In case of perceptual audio coders, these measures could not be used as an objective measure of audio quality due to the well know ' $13 \mathrm{~dB}$ miracle', but they can give a quantitative measure of proposed method against the traditional one.

\section{B. Channel modeling}

In this study we focus on the short range wireless channel $[3,4]$. We implement an interleaver and rate- $1 / 2$ convolution encoder, followed by the QPSK, 16-QAM, 64-QAM or 256-QAM modulator. The corresponding receiver functional blocks are also implemented. The short range wireless channel condition (SINR) is randomly instantiated. The joint rate control will decide on the particular modulation and audio encoding rate, depending on the channel condition for the given transmission frame.

\section{RESULTS OUTLINE}

The proposed method was compared to transmitting fixed rate audio over simulated wireless channel. Four channel types are taken into consideration with the following SINRs: 1) $0-5 \mathrm{~dB}$; 2) $5-15 \mathrm{~dB}$; 3) $15-25 \mathrm{~dB}$ and 4) $25-35 \mathrm{~dB}$. In Fig. 2 and Table 1 we present some test results. We show that for channel 4 the overall gain in audio SNR for the proposed method is approximately $11 \mathrm{~dB}$ compared to the fixed $256 \mathrm{kbps}$ coded stream. In Fig. 3, for channel 2, the overall gain in audio SNR is approximately $6.5 \mathrm{~dB}$. This shows that even under very bad channel conditions the proposed method is improving audio quality.

\begin{tabular}{|r|r|r|l|l|r|}
\hline \multicolumn{3}{|c|}{ Rate Adaptation } & \multicolumn{3}{c|}{ No Rate Adaptation } \\
\hline $\begin{array}{l}\text { Channel } \\
\text { Type }\end{array}$ & $\begin{array}{l}\text { PSNR } \\
\text { Avg }\end{array}$ & $\begin{array}{l}\text { SNR } \\
\text { Avg }\end{array}$ & Bit-rate & \multicolumn{1}{l|}{$\begin{array}{l}\text { PSNR } \\
\text { Avg }\end{array}$} & $\begin{array}{l}\text { SNR } \\
\text { Avg }\end{array}$ \\
\hline 4 & 65.84 & 40.56 & $256 \mathrm{kbps}$ & 54.83 & 29.55 \\
\hline 3 & 52.48 & 27.20 & $192 \mathrm{kbps}$ & 43.75 & 18.47 \\
\hline 2 & 38.76 & 13.48 & $128 \mathrm{kbps}$ & 32.20 & 6.92 \\
\hline 1 & 27.98 & 2.70 & $64 \mathrm{kbps}$ & 27.85 & 2.57 \\
\hline
\end{tabular}

Table 1 Average audio quality comparison for different channel conditions.

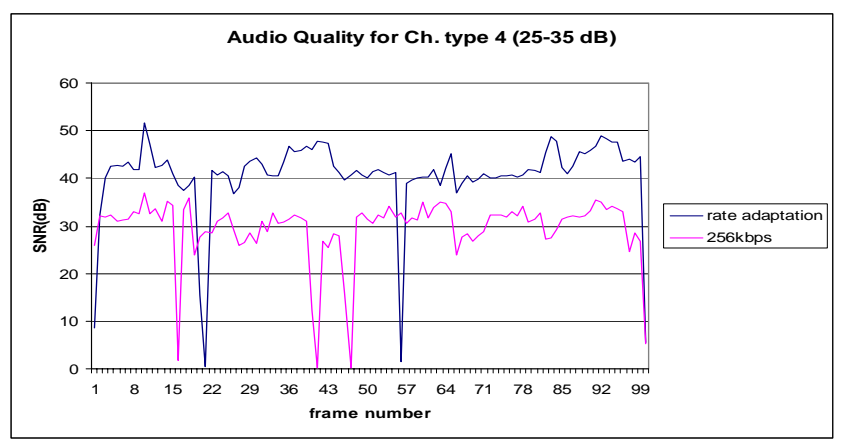

Fig. 2 Frame-level audio quality comparison of rate adaptive and non-adaptive streaming for good channel conditions.

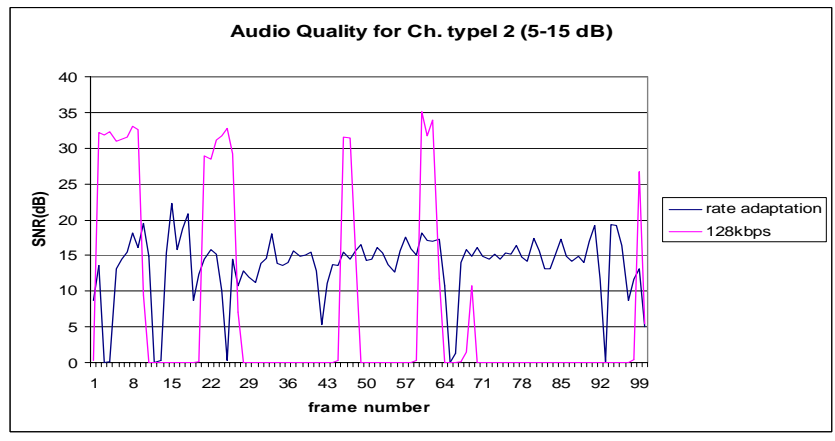

Fig. 3 Frame-level audio quality comparison of rate adaptive and non-adaptive streaming for bad channel conditions.

\section{REFERENCES}

[1] S. Khan, Y. Peng, E. Steinbach, M. Sgroi, W. Kellerer, "Applicationdriven cross-layer optimization for video streaming over wireless networks, " IEEE Communications Magazine, Vol. 44, pp. 122-130, January 2006.

[2] T. M. Cover and J.A Thomas, Elements of Information Theory, Wiley-Interscience Publication, 2001.

[3] S. S. Ghassemzadeh and V. Tarokh, "The ultra-wideband indoor path loss model," IEEE P802.15 Working Group for Wireless Personal Area Networks, 2002.

[4] V. Erceg et al. IEEE P802.11 Wireless LANs Channel Models, IEEE 802.11-03/940r4, May 2004

[5] Method for objective measurements of perceived audio quality, Recommendation ITU-R BS.1387-1, Geneva, Switzerland, 19982001. 